\title{
Modeling Virus Propagation in Peer-to-Peer Networks
}

\author{
R.W. Thommes \\ Department of Electrical and Computer Engineering \\ McGill University \\ 3480 University St, Montreal, QC, Canada H3A 2A7 \\ Email: rthomm@tsp.ece.mcgill.ca
}

\author{
M.J. Coates \\ Department of Electrical and Computer Engineering \\ McGill University \\ 3480 University St, Montreal, QC, Canada H3A 2A7 \\ Email: coates@ece.mcgill.ca
}

\begin{abstract}
The popularity of peer-to-peer (P2P) networks makes them an attractive target to the creators of viruses and other malicious code. Indeed, recently a number of viruses designed specifically to spread via P2P networks have emerged. In this paper we present a model which predicts how a P2P-based virus propagates through a network. This model is a modified version of the S-EI (Susceptible-Exposed-Infected) model from the field of epidemiology. Our model classifies each peer as falling into one of three categories based on the number of infected files it is sharing. We derive differential equations which comprise the deterministic model and examine the expected behaviour of the P2P network as predicted by these equations.
\end{abstract}

Keywords-peer-to-peer networks, viruses, epidemiology

\section{INTRODUCTION}

Several factors make peer-to-peer (P2P) networks particularly susceptible to the spreading of malicious code. The early P2P networks such as Napster could only be used to trade MP3 files, which essentially cannot contain malicious code [1]. However, contemporary P2P networks such as Kazaa / Fastrack [2] and eDonkey2000 [3] are able to disseminate executable files which may contain viruses. As P2P has increasingly entered the mainstream - the eDonkey2000 network alone typically has over 2 million users connected at any given time [4] - many users lack the technical knowledge to detect suspicious files or scan for viruses. As a result, malicious files may not be rapidly contained.

In this paper we examine how files infected with viruses propagate through a $\mathrm{P} 2 \mathrm{P}$ network. We begin by presenting a relatively simple model in Section II and describing its various parameters. Next, in Section III we derive several differential equations that govern the expected evolution of the network over time. In Section IV we analyze the steady-state behaviour of our model. Finally, in Section V we examine the effect that varying a number of model parameters has on the steadystate behaviour of the network.

\section{A. P2P Network Overview}

This section highlights the key features shared by popular P2P Networks, including Kazaa, eDonkey2000, and Gnutella [5]. Every peer connected to the network has a shared folder containing all the files the user wishes to make publicly available for download by others on the network. When a user wants to download a file, he begins by sending out a search request. Eventually he will receive back a list of files matching the search criteria. The specific manner in which this list is generated varies among the various P2P networks, but in all cases the query response is the result of the examination of the shared folders of a subset of all peers connected to the network. Once the user elects to download one of the files from the list, his client attempts to set up a connection to a peer sharing the file and begins receiving the file. Depending on the specific network, the client may attempt to simultaneously download different parts of the file from a number of peers in order to expedite the operation. P2P clients typically save new downloaded files in the shared folder - making them immediately available to other users.

A number of worms and viruses that exploit P2P networks have already surfaced. The majority of these behave in a similar fashion. Specifically, when a user downloads a file containing the virus and executes it, a number of new files containing the virus are created and placed in the client's shared directory. Some types of viruses, including Achar [6] and Gotorm [7], generate a fixed list of filenames when executed. More advanced viruses, such as Bare [8] and Krepper [9], randomly pick the list of filenames from a large pool of candidates. 


\section{B. Related Work}

The advent of mathematical Epidemiology - the field of biology which models how diseases spread in a population - is generally credited to McKendrick and his seminal 1926 paper [10]. Previous work in applying epidemiology to modeling how computer viruses and other malware spreads between machines dates back to the early 1990s: Kephart and White published a paper [11] on the topic in 1991. More recently, Zou et al. utilized epidemiology to model the spread of the Code Red across the Internet [12].

There have been a number of recent papers which model file propagation in $\mathrm{P} 2 \mathrm{P}$ networks. Two notable examples include a 2005 paper by Dumitriu et al. [13] which models the spread of polluted files in P2P networks, and a 2004 paper by Qiu and Srikant [14] which models the performance of the BitTorrent P2P protocol.

\section{MODEL Description}

The intent of our model is to predict the expected behaviour of a virus which spreads through a P2P network in the form of malicious code embedded in executable files shared by peers. We make the simplifying assumption that all users download files to their shared folder. We are not concerned with the transfer of media files which cannot contain malicious code, and do not model them. Note that we use the term user in this paper to refer to a person using a $\mathrm{P} 2 \mathrm{P}$ client program. The term peer is used to collectively refer to a P2P client and the user directing its behaviour.

This model classifies all peers as falling into one of three classes: Susceptible, Exposed, or Infected:

Susceptible - Peers that are not sharing any infected files, but are at risk of downloading infected files. The number of peers in this category at time $t$ is denoted by $S(t)$.

Exposed - Peers that have downloaded one or more infected files, but have not executed them. The number of peers in this category at time $t$ is denoted by $E(t)$.

Infected - Peers that have executed an infected file. Upon execution, a total of of $c$ infected files reside in the peer's shared folder. The number of peers in this category at time $t$ is denoted by $I(t)$.

An Infected client may be detected by the user, who will then proceed to remove all the infected files, thereby returning the state of the peer to Susceptible. At all times, every one of the $N$ peers making up the network falls into one of the three categories. Thus, for all values of $t, N=S(t)+E(t)+I(t)$.

We assume that the total number of uninfected files in the network is fixed at $M$. The total number of infected files at time $t$ is given by $K(t)$. The expected proportion of infected files in the network, $q(t)$, is therefore $q(t)=\frac{K(t)}{K(t)+M}$. When a user downloads a file, we assume the probability of choosing an infected file will be dependent on the prevalence of infected files in the network. We model this dependence as being time-invariant in the sense that it only depends on the current value of $q(t)$, and denote the function mapping $q(t)$ to the probability of downloading an infected file as $f\{q(t)\}$. In Section IV we set $f\{q(t)\}=\alpha q(t)$ to simplify our analysis. However, we concede that this may not necessarily reflect the download behaviour in $\mathrm{P} 2 \mathrm{P}$ networks in an accurate manner.

There are three distinct events that may occur in the network which affect one or more of the time-varying variables described above. These events include a peer downloading a file from another, a peer executing a shared file, and an Infected peer recovering. The average rates at which each of these events occurs are governed by three parameters:

$\lambda_{S}$ : Average rate, in files per minute, at which each peer downloads new files (this includes time spent searching and setting up the connection to another peer).

$\lambda_{E}$ : Average rate, in files per minute, at which each peer executes shared files. We assume that a peer executes files in the order in which they are downloaded.

$\lambda_{R}$ : Average rate, in "recoveries per minute", at which Infected peers recover. A recovery occurs when all infected files are removed, returning the peer state to Susceptible.

\section{MODEL EQUATIONS}

Table I summarizes which time-varying variables are affected by each of the three events that may occur in the network:

The state progression for all peers in our model is $S \rightarrow E \rightarrow I \rightarrow S \ldots$. We now derive the differential equations that govern the evolution of our P2P model.

\section{A. Rate at which number of Infected peers change}

When an Infected peer recovers, the number of Infected peers decreases by one. Recoveries occur at rate 


\begin{tabular}{|c|c|}
\hline Event & Variables Affected \\
\hline \hline File downloaded & $q(t), S(t), E(t)$ \\
\hline File executed & $q(t), E(t), I(t)$ \\
\hline Peer recovers & $q(t), I(t), R(t)$ \\
\hline
\end{tabular}

TABLE I. Variables potentially affected by each possible event

$\lambda_{R} I(t)$. When an Exposed peer executes an infected file, the number of Infected peers increases by one. Since files are executed in order of download, the file executed by an Exposed peer will always be the infected file which it had downloaded to become Exposed. This occurs at a rate of $\lambda_{E} E(t)$. Therefore,

$$
\frac{d I(t)}{d t}=-\lambda_{R} I(t)+\lambda_{E} E(t)
$$

\section{B. Rate at which number of Exposed peers change}

The rate at which the number of Exposed peers decreases due to infection is given by the negative of the second term in (1). The rate at which previously Susceptible peers become Exposed is dependent on the aggregate rate at which they download files: $\lambda_{S} S(t)$, multiplied by the probability that a downloaded file is infected: $f\{q(t)\}$. The overall rate is therefore:

$$
\frac{d E(t)}{d t}=-\lambda_{E} E(t)+\lambda_{S} S(t) f\{q(t)\}
$$

\section{Rate at which number of Susceptible peers change}

This is governed by the negatives of the the first term in (1) and the second term in (2):

$$
\frac{d S(t)}{d t}=-\lambda_{S} S(t) f\{q(t)\}+\lambda_{R} I(t)
$$

D. Rate at which number of infected files in the network changes

There are three events which result in a change in the number of infected files in the network: a peer downloads an infected file, an Exposed peer becomes Infected, and an Infected peer recovers. We assume that all downloaded files are executed, and that a peer does not download any additional files prior to executing the most recently downloaded file.

Peers cannot share more than one copy of a file with the same name. If the number of unique filenames is limited to $c$, only Susceptible peers can download infected files. Exposed peers do not download any additional files before becoming Infected, and Infected peers are sharing all $c$ possible infected files. Thus, the rate of change due to downloads is $S(t) \lambda_{S} f\{q(t)\}$.

An Exposed peer always has one infected file before becoming Infected, meaning in all cases $c-1$ new infected files are created when an Exposed peer becomes Infected. The rate of change is thus $E(t) \lambda_{S}(c-1)$.

An Infected peer will always share $c$ files, so a recovery results in a reduction of $c$ infected files. The rate is therefore $-I(t) \lambda_{R} c$. The overall rate of change of $K$ is therefore:

$$
\frac{d K(t)}{d t}=S(t) \lambda_{S} f\{q(t)\}+E(t) \lambda_{E}(c-1)-I(t) \lambda_{R} c
$$

We note that if the names of generated files are chosen from a pool of names $>>c$, Infected peers can continue to download infected files and the above equation does not hold. However, we will not consider this case in any additional detail in this paper.

\section{STEAdy-STATE Behaviour}

If the $\mathrm{P} 2 \mathrm{P}$ network reaches a steady-state equilibrium by some time $t=T$, then $\frac{d E(T)}{d t}=\frac{d I(T)}{d t}=\frac{d S(T)}{d t}=0$. Defining $\tilde{E}, \tilde{I}, \tilde{S}$, as the steady-state values of, respectively, $E(t), I(t)$, and $S(t)$, Equation (1) implies that:

$$
\tilde{I}=\tilde{E} \frac{\lambda_{E}}{\lambda_{R}}
$$

If we define $\tau$ and $\mu$ as, respectively, the expected number of infected files each Exposed and Infected peer is sharing in steady-state, then $\tilde{q}$, the proportion of infected files in steady-state may be expressed as:

$$
\tilde{q}=\frac{\tilde{E} \tau+\tilde{I} \mu}{M+\tilde{E} \tau+\tilde{I} \mu}
$$

Substituting (5) into (6) provides:

$$
\tilde{q}=\frac{\tilde{E}\left(\tau \lambda_{R}+\mu \lambda_{E}\right)}{M \lambda_{R}+\tilde{E}\left(\tau \lambda_{R}+\mu \lambda_{E}\right)}
$$

If $f\{\tilde{q}\}>0$, equation (2) implies that, in steady state:

$$
\tilde{S}=\tilde{E} \frac{\lambda_{E}}{\lambda_{S} f\{\tilde{q}\}}
$$

Since $\tilde{S}=N-\tilde{I}-\tilde{E}$, equation (5) can be utilized to express $N$ as:

$$
\tilde{S}=N-\tilde{E}\left(1+\frac{\lambda_{E}}{\lambda_{R}}\right)
$$

If $f\{q(t)\}$ is proportional to $q(t): f\{q(t)\}=\alpha q(t)$, we may obtain a closed-form expression for $\tilde{E}$ by substituting (7) into (8), equating with (9), and solving for $\tilde{E}$ :

$$
\tilde{E}=\frac{\lambda_{R} \alpha\left(N \lambda_{S}\left(\mu \lambda_{E}+\tau \lambda_{R}\right)-M \lambda_{E} \lambda_{R}\right)}{\left(\tau \lambda_{R}+\mu \lambda_{E}\right)\left(\lambda_{S} \alpha\left(\lambda_{R}+\lambda_{E}\right)+\lambda_{E} \lambda_{R}\right)} ; \tilde{q}>0
$$


The expression for $\tilde{I}$ follows trivially from (10) and (5):

$$
\tilde{I}=\frac{\lambda_{E} \alpha\left(N \lambda_{S}\left(\mu \lambda_{E}+\tau \lambda_{R}\right)-M \lambda_{E} \lambda_{R}\right)}{\left(\tau \lambda_{R}+\mu \lambda_{E}\right)\left(\lambda_{S} \alpha\left(\lambda_{R}+\lambda_{E}\right)+\lambda_{E} \lambda_{R}\right)} ; \tilde{q}>0
$$

If $\tilde{q}=0$, it follows from (6) that $\tilde{E}=\tilde{I}=0$. It is of interest to consider Equation (11) as it approaches 0. In the limiting case, approached from above, we have the equality

$$
N \lambda_{S}\left(\mu \lambda_{E}+\tau \lambda_{R}\right)=M \lambda_{E} \lambda_{R}
$$

Since we assume that all downloaded files are eventually executed, it follows that $\lambda_{E}=\lambda_{S}$ if we consider these rates to be averaged over a sufficiently long interval. Under this assumption, (12) provides the following minimum average recover rate, $\lambda_{R}^{\min }$ in order for all infected files to eventually be removed from a P2P network:

$$
\lambda_{R}^{\min }=\frac{N \mu \lambda_{E}}{M-N \tau} ; M>N \tau
$$

This equation indicates that, if $f\{q(t)\}=\alpha q(t)$, then $\lambda_{R}^{m i n}$ is a linearly increasing function of $\lambda_{E}$.

\section{Results}

In this section we provide some examples of virus behaviour in P2P network predicted by our model. The value of $\tau$ is 1 and $\mu=c$, which follows from the discussion in Section III-D. Figure 1 illustrates how the number of peers falling into each of the three categories evolve over time, and eventually reach a steady state. In this case, $\lambda_{E}=\lambda_{S}=3.47 \times 10^{-3}$ files per minute, which corresponds to 5 downloads per day. The average time for a peer to recover is 24 hours, meaning $\lambda_{R}$ is $6.94 \times 10^{-4}$. The number of peers, $N$, is 2 million and there are 60 million clean files $M$. This example makes use of the model in which the number of unique possible files is limited to $c$, and $c$ is 10 . Finally, $f\{q(t)\}=$ $0.5 q(t)$. Initially, there are 10000 Exposed peers, each sharing one infected file.

In Figure 2 we examine the number of infected peers in the network when varying the initial number of infected peers. After about 700 hours, the three networks reach the same steady-state. This is also the behaviour implicitly predicted by equation 11 , since it is independent of any initial condition (as long as at least one infected file initially exists in the network).

Figures 3, 4, and 5 examine the effect that, respectively, $c, \alpha$, and $\lambda_{E}$ have on the steady-state number of infected peers and the proportion of infected files. The relationship between the number of infected peers and

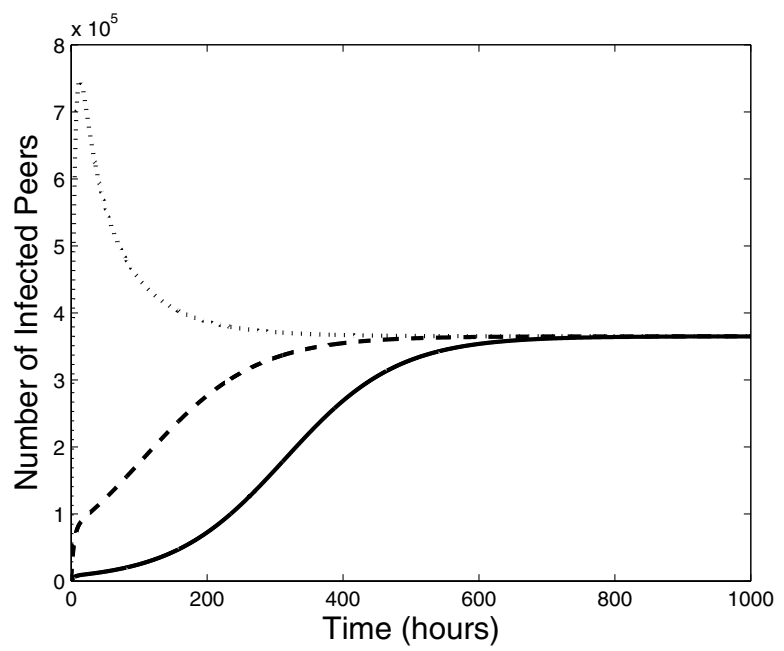

Fig. 2. The number of infected peers vs. time for different initial conditions. The solid line corresponds to 10000 infected files initially in the network, the dashed line: 100000 initial infected files, the dotted line: 1000000 initial infected files

files and both $\alpha$ and $\lambda_{E}$ may be approximated as linear over the ranges considered, whereas the dependence on $c$ suggests a log-function.

\section{CONCLUSION}

We have presented a model of how infected files spread in a P2P network, and derived expressions for the steady-state behaviour in the case where the probability of a peer downloading an infected file is proportional to the prevalence of infected files in the network. In future work we will derive a function mapping file popularity to download rates in a manner that more closely mirrors user behaviour in an actual P2P network and model the dynamics of a virus that can choose file names from a pool much larger than $c$.

\section{REFERENCES}

[1] F-Secure, "F-secure hoax information pages: Mp3 virus," http://www.f-secure.com/hoaxes/mp3.shtml, 1998.

[2] "Kazaa," www.kazaa.com.

[3] "Edonkey2000," www.edonkey2000.com.

[4] "eDonkey2000 server list," http://ocbmaurice.no-ip.org/slist /serverlist.html.

[5] "Gnutella protocol development," http://rfc-gnutella. sourceforge.net.

[6] Viruslist.com, "P2p-worm.win32.achar.a," http://www.viruslist. com/en/viruses/encyclopedia?virusid=23893, May 2003.

[7] Symantec, "W32.hllw.gotorm," http://securityresponse. symantec.com/avcenter/venc/data/w32.hllw.gotorm.html, August 2003.

[8] Viruscan, "W32/bare.worm," http://www.virus-scan-software .com/latest-virus-software/latest-viruses/w32bare-worm.shtml, 2003.

[9] Sophos, "Sophos virus analysis: Troj/krepper-g," http://www .sophos.com/virusinfo/analyses/trojkrepperg.html, July 2004. 


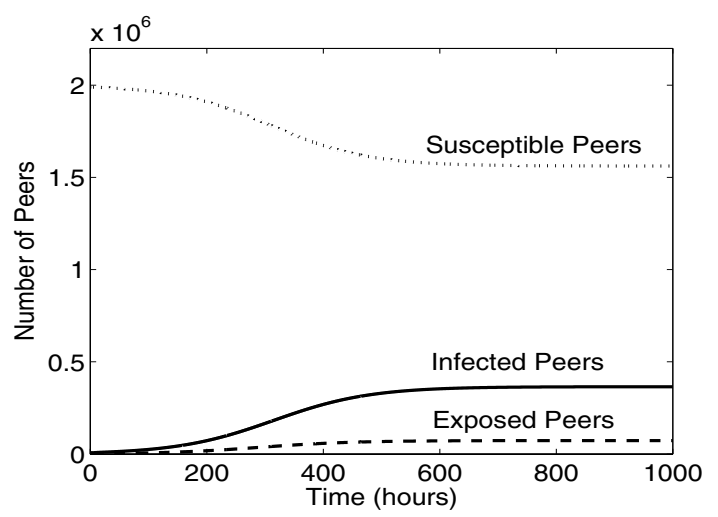

(a) The number of peers in each group

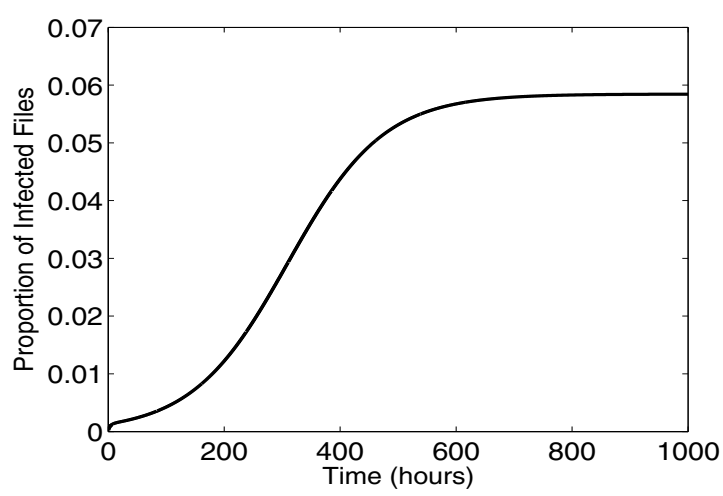

(b) The proportion of infected files

Fig. 1. Example of the dynamic behaviour of a P2P network exposed to a virus. The network reaches steady-state after about 600 hours.
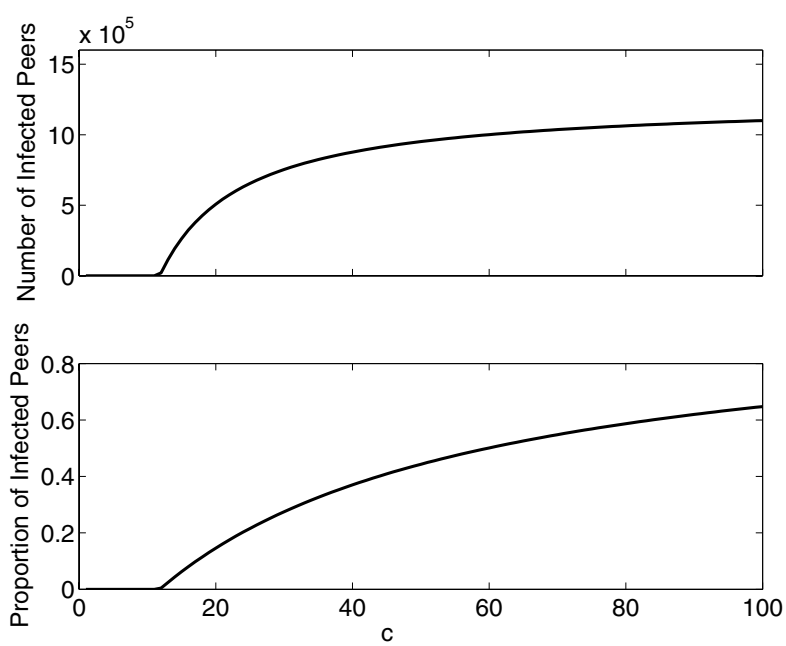

Fig. 3. Examining the effect of $c$ on the steady-state number of infected peers and infected files.
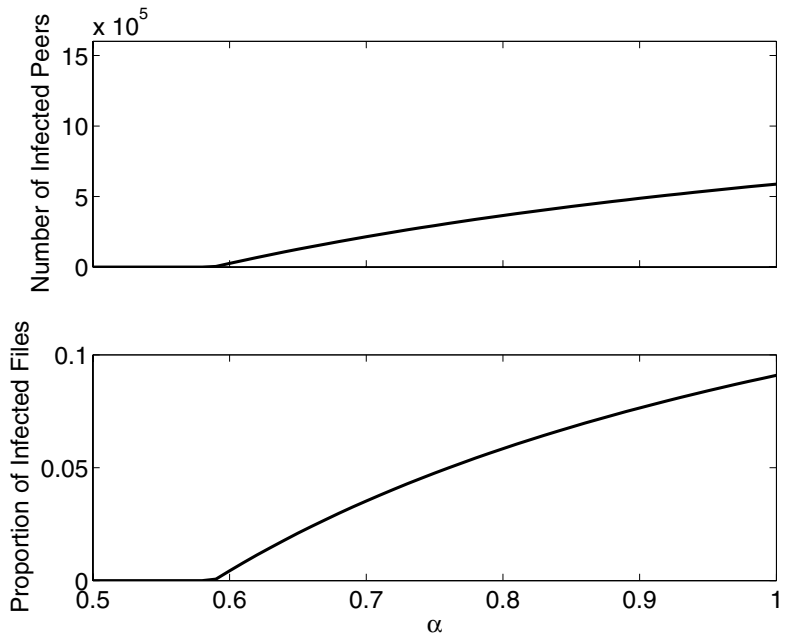

Fig. 4. Examining the effect of $\alpha$ on the steady-state number of infected peers and infected files.
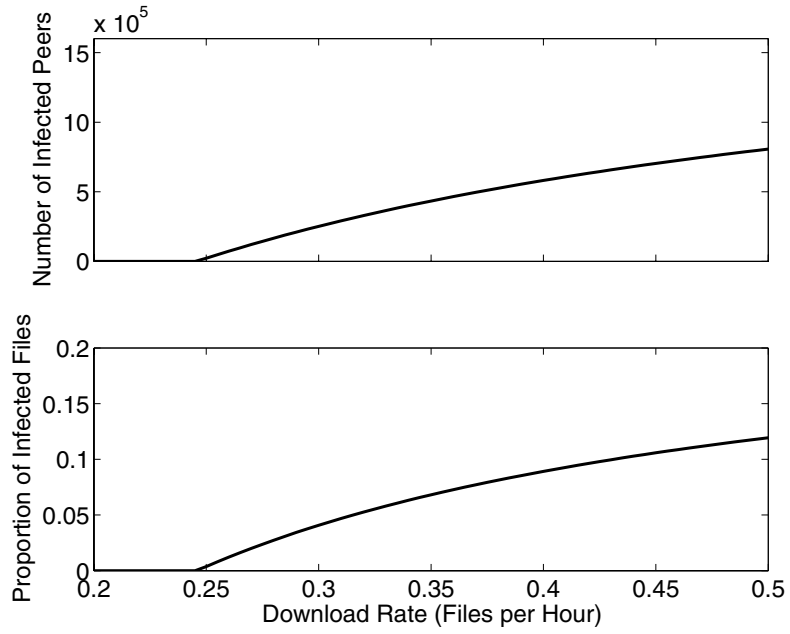

Fig. 5. Examining the effect of $\lambda_{E}$ on the steady-state number of infected peers and infected files.

[10] A.G. McKendrick, "Applications of mathematics to medical problems," Proc. Edinb. Math. Soc., vol. 44, pp. 98-130, 1926.

[11] J.O. Kephart and S.R. White, "Directed-graph epidemiological models of computer viruses," in Proc. IEEE Symp. Security and Privacy, Oakland, CA, May 1991.

[12] C.C. Zou, W. Gong, and D. Towsley, "Code red worm propagation modeling and analysis," in Proc. ACM Conf. Computer and Comm. Soc., Washington DC, Nov 2002.

[13] D. Dumitriu, E. Knightly, A. Kuzmanovic, I. Stoica, and W. Zwaenepoel, "Denial-of-service resilience in peer-to-peer file-sharing systems," in Proc. ACM Sigmetrics, Banff, Canada, June 2005.

[14] D. Qiu and R. Srikant, "Modeling and performance analysis of bittorrent-like peer-to-peer networks," in Proc. ACM Sigcomm, Portland, OR, Aug. 2004. 